\title{
Characterization of Polylactic Acid Colloids for In Situ Bioremediation
}

Ashley N. Westbrook

Summer Student

Florida A\&M Universtiy

July 2003

Idaho National Engineering and Environmental Laboratory Bechtel BWXT Idaho, LLC 
INEEL/EXT-03-0954

\title{
Characterization of Polylactic Acid Colloids For \\ In Situ Bioremediation
}

\author{
Ashley N. Westbrook \\ Summer Student \\ Florida A\&M University
}

July 2003

Idaho National Engineering and Environmental Laboratory

Idaho Falls, Idaho 83415

Prepared for the

U.S. Department of Energy

Assistant Secretary

Under DOE Idaho Operations Office

Contract DE-AC07-99ID13727 


\title{
CHARACTERIZATION OF POLYLACTIC ACID COLLOIDS FOR IN SITU BIOREMEDIATION
}

\author{
Ashley N. Westbrook \\ Summer Student \\ Idaho National Engineering and Environmental Laboratory \\ Idaho Falls, Idaho
}

\section{INTRODUCTION}

Groundwater contamination is a widespread problem. As human activities generate chemical wastes, we seek quick, yet not always environmentally friendly, ways to dispose of them. Often, chemicals are dumped into waste containers and buried. Unfortunately, these chemicals may spill or leak, seep deeper into the ground, and eventually reach groundwater. Chlorinated organic solvents such as trichloroethene, tetrachloroethane, and chloroform are common contaminants in groundwater [1]. These solvents originate from chemical spills, tank leaks, and waste disposal practices [2]. Such contaminants are harmful to humans, and need to be removed to restore clean groundwater.

There are two types of methods to clean chemically contaminated soil and groundwater: ex situ and in situ. Both types have advantages and disadvantages. In situ remediation avoids the cost of removing the contaminated material and has a lower risk of worker exposure to the contaminants. One in situ technique that shows promise is bioremediation, which uses microorganisms to anaerobically degrade contaminants. In bioremediation in situ, the microorganisms that are already present in the ground utilize nutrients that are injected into the ground to metabolize the contaminants into nonhazardous materials. The nutrients serve as a carbon source for the microbes. Though this method has been used with some success, there have been problems with too rapid release of nutrients to the microbes. In these cases, the microbes multiplied too rapidly and caused biofouling. This can occur when the nutrient content of the bioremediation solution is too high, as when lactate or lactic acid is the nutrient. Lactic acid is typically produced in carbohydrate matter by fermentation, and is used mainly in the food and pharmaceutical industries [3]. Because it has been found difficult to uniformly distribute lactate solutions in a contaminated area due to biofouling, it was suggested that polylactic acid, in colloidal form, be used instead.

Polylactic acid (PLA) is a polymer of lactic acid, $\mathrm{C}_{3} \mathrm{H}_{6} \mathrm{O}_{3}$ [3]. PLA is used in medicine and agriculture. In medicine, PLA is used in sutures because it degrades within the body after the incision has healed. In agriculture, PLA is used in combination with polyglycolic acid for the release of chemicals [4]. We expect PLA to degrade in the ground at a rate that allows bioremediation to occur as intended.

Currently, bioremediation of chlorinated solvents involves injecting nutrientcontaining solvents into the ground to induce the expected activity of the microbes. The solution must have a proper amount of nutrients for the microbes to metabolize so that 
they can continue to degrade the contaminants. If too much nutrient is available, and the microbes multiply too fast and clog the injection point, the solution may not reach the area where the contaminants are. One way to slowly release the nutrients for bioremediation is to package them as colloids. Colloids are particles that are less than one micrometer in size, with surface chemical properties that allow them to remain suspended in water and, therefore, to travel with water. Our research addresses the problem of packaging nutrients as colloids to support bioremediation.

\section{OBJECTIVE OF EXPERIMENTS}

We investigated and characterized colloids to aid in the fabrication of a colloid that has the nutrient content and mobility needed for bioremediation. The first concern was that the nutrients must be in a colloidal form that will not flocculate. When flocculation occurs, particles stick to each other to form one large clump, which will eventually become too dense to float in water. If the particles are too dense to remain in suspension, they will be inefficient in flowing through the groundwater and distributing the nutrients. With such questions in mind, we began to investigate and create an "ideal colloid."

\section{EXPERIMENTS}

Several experiments were conducted to synthesize colloids that would be useful in bioremediation. Each experiment brought us one step closer to creating the intended colloids. Some experiments were conducted to synthesize the colloids. In these experiments, we used data from past projects to gather the materials to make the colloids. Once the colloids were made, a series of experiments were conducted to characterize them. For bioremediation to be successful, we needed to know how the colloids would move through the ground before we actually injected them into the ground. For this reason, we used simulated ground to investigate the activity of the colloids.

\section{Synthesis}

In the first series of experiments, $75.0 \mathrm{~mL}$ of tetrahrdrofuran ([THF] formula weight 72.11; Fisher Chemicals) was mixed with $3.0 \mathrm{~g}$ of Poly (L-Lactic Acid) (molecular weight 2,000; Polysciences), under gentle heat of about $40^{\circ} \mathrm{C}$ to dissolve the PLA. Next, the polymer and $0.0025 \mathrm{~g}$ of Fluorescein labeled Polystyrene (molecular weight 7,700; Polysciences, Inc.) was added to the THF/PLA solution, and mixed until a uniform solution was obtained. $5.0 \mathrm{~mL}$ of the polymer were then loaded into a 5 -mL syringe. The syringe was then loaded into a syringe pump (Model 341A, Sage Instruments). One end of a plastic tube was placed over the needle of the syringe, and the other tube was placed through the side of the horn (Figure 1) of the sonic dismembrator (Model 500; Fisher Scientific) and out at the end of the horn. On the other side of the horn, the tube from the recirculating pump (Easy Load Master Flex, Model 7518-10; Cole Parmer Instrument Company) ran from the side of the sonic dismembrator's horn, around the rotor, with the 
other end in a beaker of deionized water (Figure 1). A stir bar lay in the bottom of the beaker, and was stirred by a magnetic stirrer (Thermolyne $\operatorname{Cimarec}^{\mathrm{R}} 2$, model number SP6925).

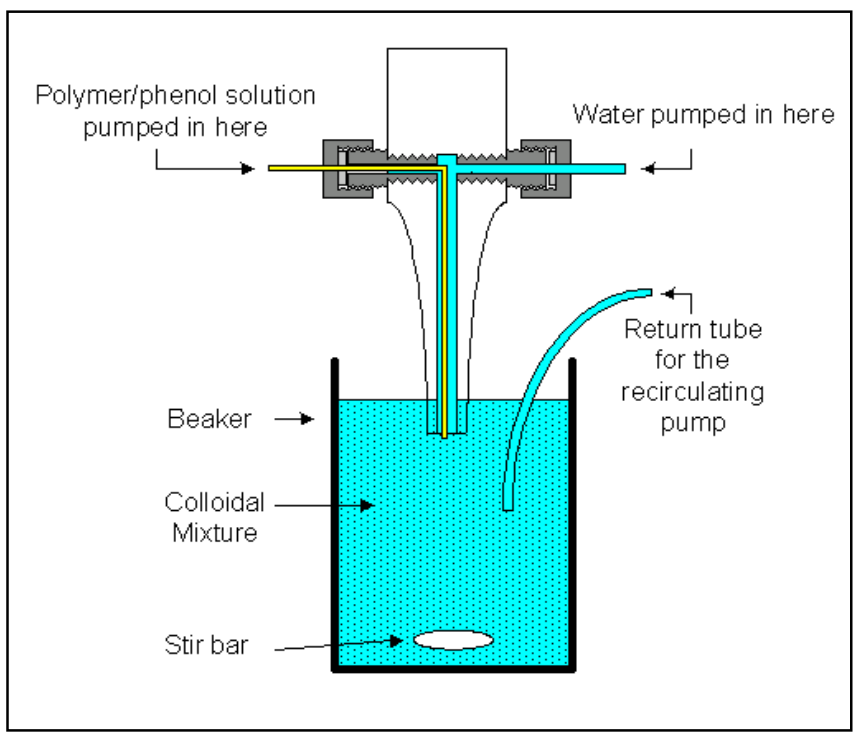

Figure 1.

The set up of the apparatus used to fabricate the PLA colloids.

The syringe pump's settings allowed variations in the rate at which the syringe emitted the polymer in $\mathrm{mL} / \mathrm{h}$ and $\mathrm{mL} / \mathrm{min}$. Several trials were run with various speeds of emission to identify the speed that produced the smallest colloids. We emitted the solution at a rate of $1.7,1.0,0.51,0.24$, and $0.10 \mathrm{~mL} / \mathrm{min} ; 0.51 \mathrm{~mL} / \mathrm{min}$ yielded the smallest colloids (Table 1).

\begin{tabular}{|l|l|l|}
\hline Volume of water $(\mathrm{mL})$ & Rate of emission $(\mathrm{mL} / \mathrm{min})$ & Mean size of particles $(\mathrm{nm})$ \\
\hline 500 & 1.7 & 635.2 \\
\hline 500 & 1.0 & 594.3 \\
\hline 500 & 0.51 & 495.0 \\
\hline 500 & 0.4 & 594.2 \\
\hline 500 & 0.10 & 629.3 \\
\hline
\end{tabular}

Table 1.

The size of the colloids at various speeds of emission of solution into $500 \mathrm{~mL}$ of deionized water.

The volume of deionized water into which the solution was released was another variable. $5.0 \mathrm{~mL}$ of solution was released into 50,100,200, 500, and $1000 \mathrm{~mL}$ of water to determine which volume would yield the smallest particles (Table 2). When the experiment was repeated, the smallest particles were produced when $5.0 \mathrm{~mL}$ of solution was emitted into $1000 \mathrm{~mL}$ of deionized water at a rate of $0.51 \mathrm{~mL} / \mathrm{min}$. These parameters were used to create Batch 2 and Batch 3. 


\begin{tabular}{|l|l|l|}
\hline Volume of water $(\mathrm{mL})$ & Rate of emission $(\mathrm{mL} / \mathrm{min})$ & Mean size of particles $(\mathrm{nm})$ \\
\hline 50 & 0.51 & 730.9 \\
\hline 100 & 0.51 & 683.2 \\
\hline 200 & 0.51 & 947.7 \\
\hline 500 & 0.51 & 495.0 \\
\hline 1000 & 0.51 & 455.0 \\
\hline
\end{tabular}

Table 2.

The size of the colloids for various water volumes.

The next step in PLA colloid synthesis involved separating the THF and unincorporated dye from the PLA colloids suspension. For this, a centrifuge (IEC Centra MP4; model number MP4R 24382674; International Equipment Company) was used. Approximately $40 \mathrm{~mL}$ of suspension was poured into two plastic centrifuge tubes $\left(50 \mathrm{~mL}\right.$; Fisherbrand $\left.{ }^{\mathrm{R}}\right)$. The two tubes were then weighed to within $0.1 \mathrm{~g}$ difference, loaded into the centrifuge, and spun at 6500 RPMs for 15 min. After spinning, the tubes were removed from the centrifuge and examined. A pale fluorescent yellow powder seemed to stick to the bottom corner of the tube. The liquid was then decanted from the sticky powder by gently pouring the liquid into a waste container, with care taken not to disturb what lay in the bottom of the tube. This process was repeated until all of the solution had been spun and decanted. At this point, about $50 \mathrm{~mL}$ of deionized water was added to each tube and the colloids were re-suspended using sonication and were then spun at 6500 RPMs for $15 \mathrm{~min}$ to isolate the product. The water was then decanted with the same procedure used previously. This process was repeated twice. After the last decantation, about $10 \mathrm{~mL}$ of deionized water was added to each tube. The tubes were then shaken and placed in a small sonicator (Fisher Scientifics; model IS 20) for about $30 \mathrm{~s}$ to remove the product from the sides of the tube. These solutions were combined in a glass container and named Batch 1 . Two additional batches were later made with the same procedure, and named Batch 2 and Batch 3.

\section{Characterization}

A small sample of the Batch 1 solution was placed in a cuvette and loaded into a particle size analyzer (Coulter N4 Plus; Beckman Coulter). The analyzer measured the size of the particles in nanometers, and gave a reading of the mean size of the particles. Images of freeze-dried colloids were processed by a scanning electron microscope (Figure 2). 


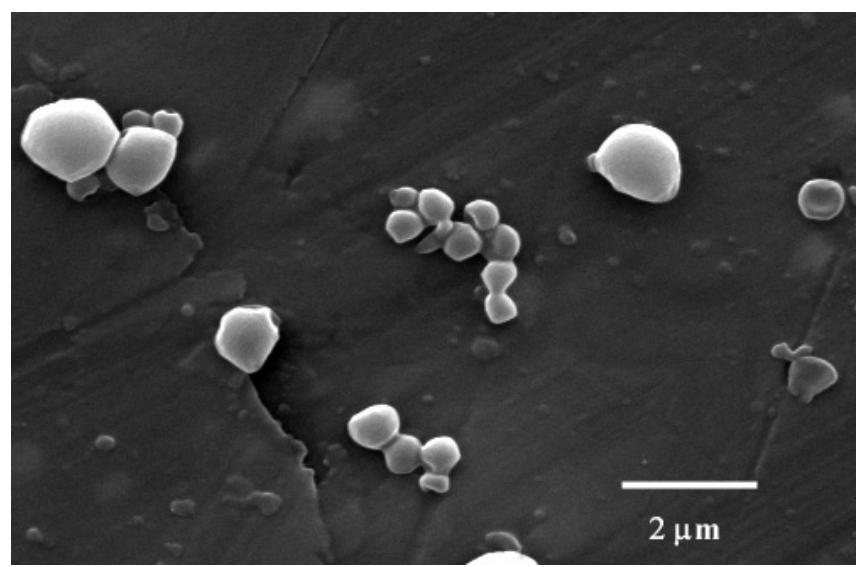

\section{Figure 2}

This image of the colloids was produced by a scanning electron microscope.

After the size of the colloids had been determined to be $455 \mathrm{~nm}$, the fluorescence of the particles was investigated. A 2-mL sample of the solution was placed in a glass cuvette, and then loaded into a fluorescence spectrometer (Fluorolog Spectra CQ, ISA Instruments). The excitation wavelength was set at $450 \mathrm{~nm}$, with an emission wavelength of $650 \mathrm{~nm}$. An unfiltered sample of Batch 1 and two samples of Batch 1 filtered at $1.0 \mu \mathrm{m}$ and $0.2 \mu \mathrm{m}$ were loaded into the spectrometer. The readings of the fluorescence spectrometer are shown in Figure 3. The unfiltered batch shows the highest peak on the graph, which indicated the highest counts. This means that the fluorescent dye was being fluoresced and that those particles were being detected. This could have been due to the free dye in the suspension, or the colloids fluorescing. When Batch 1 was filtered through a $1.0-\mu \mathrm{m}$ filter, the counts were significantly lower, as indicated by the broad region of the graph. This indicates that the particles larger than $1.0 \mu \mathrm{m}$ may have been responsible for the higher counts. When the solution was filtered through a $0.2-\mu \mathrm{m}$ filter, the counts were even lower and, when graphed, had a sharper peak than the other two readings. This indicates that anything detected at this point was either free dye, or les than $0.2 \mu \mathrm{m}$.

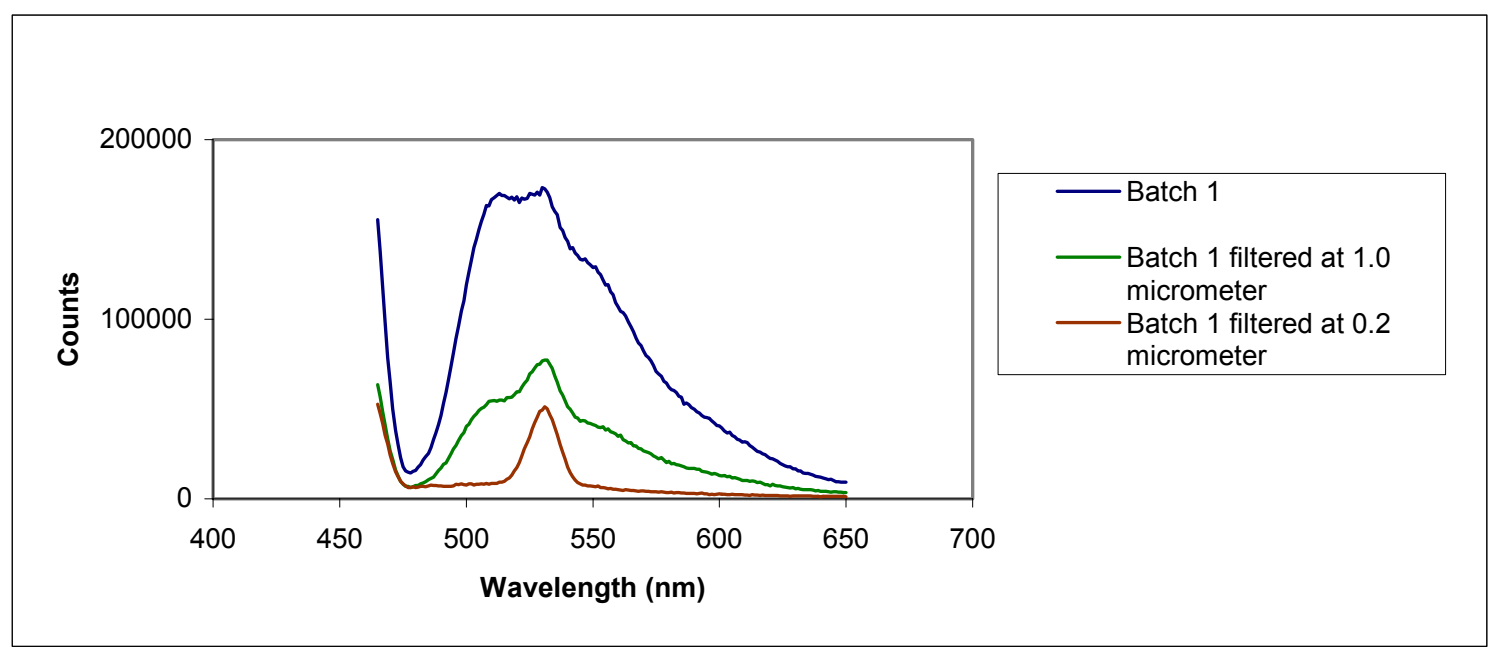

Figure 3.

The wavelengths of the colloids in Batch 1. The peaks indicate the detection of florescent properties in the colloids. 
The next characterization test measured electrophoretic mobility or zeta potential of the colloids. Zeta potential is a measure of surface charge. It can be related to the shear plane, or slipping plane of a surface, which is an imaginary surface separating the thin layer of liquid bound to the solid surface, which has elastic behavior, from the rest of the liquid, which has viscous behavior. The surface charge of a particle is important in predicting the coagulation (clumping) of the particles, and influences how fast or slowly sedimentation occurs. If the absolute value of the zeta potential is above $50 \mathrm{mV}$, the colloids disperse in a stable manner as a result of electrostatic repulsion; when the zeta potential is close to zero, coagulation occurs more rapidly [5]. The zeta potential of Batch 1, measured using a zeta potential analyzer (Delsa 440 SX, Beckman Coulter), had an average of $-47.5 \mathrm{mV}$. Thus, the colloids were stable in simulated groundwater, and will flocculate at a slow pace.

\section{Mobility Determination}

We investigated the movement of the colloids through the ground using a column packed with basalt. Fractured basalt was chosen because of its abundance at the INEEL, and because we wanted to see if dispersed PLA colloids could pass through fractured basalt. The column, shown in Figure 4, consists of an acrylic cylinder (5.127-cm high, $5.1-\mathrm{cm}$ inner diameter, $105.8-\mathrm{cm}^{3}$ volume) with acrylic end plugs (7.6-cm diameter, 1.4$\mathrm{cm}$ high. The end plugs were lined with two 500- $\mu \mathrm{m}$ mesh macro filters (Spectra/Mesh macro porous filters, Spectra), and one 210- $\mu \mathrm{m}$ mesh macro filter. Each acrylic end plug has hole for tubing. Screws hold the end plugs on the cylinder. An intravenous bag was positioned above the column with a tube running from the bag to the top of the column. An infusion pump (AVI 200A Infusion Pump, 3M) pumped fluid from the bag to the column at a rate of $10 \mathrm{~mL} / \mathrm{hr}$. After passing through the column the fluid was collected in 5.0-mL sample vials via an auto collector.

To perform the experiment, the column was loaded with $537.2 \mathrm{~g}$ of fractured basalt, and then flushed with deionized water for $2 \mathrm{~h}$ to clean the system. Then a salt solution was prepared to run through the column. The salt solution simulated the salt compounds present on soil and ensured that the colloids stayed in suspension. From earlier experiments with the salt solution, it was determined that the solution must have a concentration of $1 \mathrm{mmol} / \mathrm{L}$. $1 \mathrm{~L}$ of deionized water was added to the sodium chloride, and the solution was shaken to mix. The solution was then transferred to an intravenous bag and run through the column for $2 \mathrm{~h}$.

Trials were then run to determine the dilution factor needed to keep the colloids in suspension. It was known that the colloids had to have counts of $1.0 \times 10^{6}$ with a size around $500 \mathrm{~nm}$ to remain in suspension. The dilution factor was determined to be 10 for Batch 3, which meant that 10 times more sodium chloride solution than PLA solution was needed to keep the colloids in suspension. $50.0 \mathrm{~mL}$ of PLA solution was added to $500 \mathrm{~mL}$ of sodium chloride solution, and then loaded into an intravenous bag to be passed through the column. The suspension dripped into test tubes in an interval sampler (IS-95 
Interval Sampler, Spectrum Chromatography). The apparatus was programmed such that $5.0 \mathrm{~mL}$ samples were collected. When all of the suspension had been run through the column, the particle sizes of the samples in the test tubes were measured. The particle concentration was measured semiquantitatively using the $90^{\circ}$ detector signal on the Coulter N4. The readings from the Coulter N4 gave the counts of the particles, which indicate how well the colloids passed through the column.

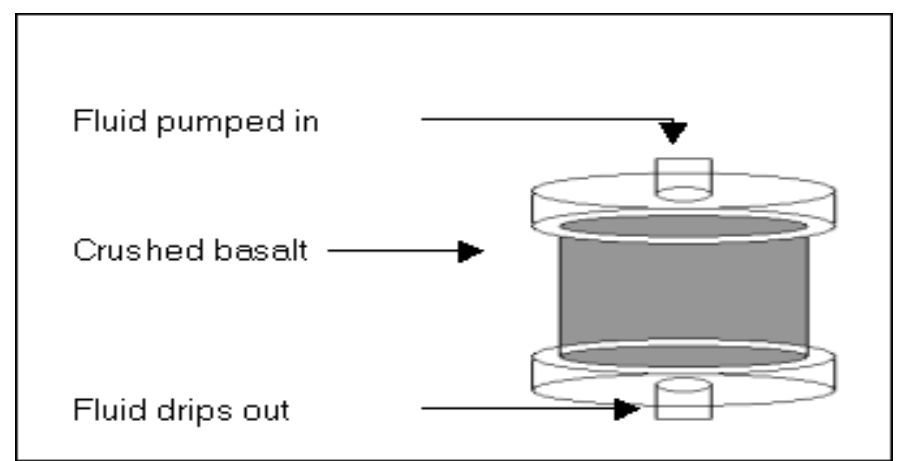

\section{Figure 4.}

The setup of the basalt column that the water and colloids were passed through.

\section{RESULTS}

The particle size analyzer and fluorescence spectrometer results showed that the particles collected had counts between $1 \times 10^{4}$ and $4.5 \times 10^{4}$, which are significantly lower than the $1 \times 10^{6}$ counts of the colloidal suspension. The results of the Coulter N4 displayed much lower counts than that expressed before the column experiment (Figure 5). It was determined that less than $10 \%$ of the colloids passed through the column.

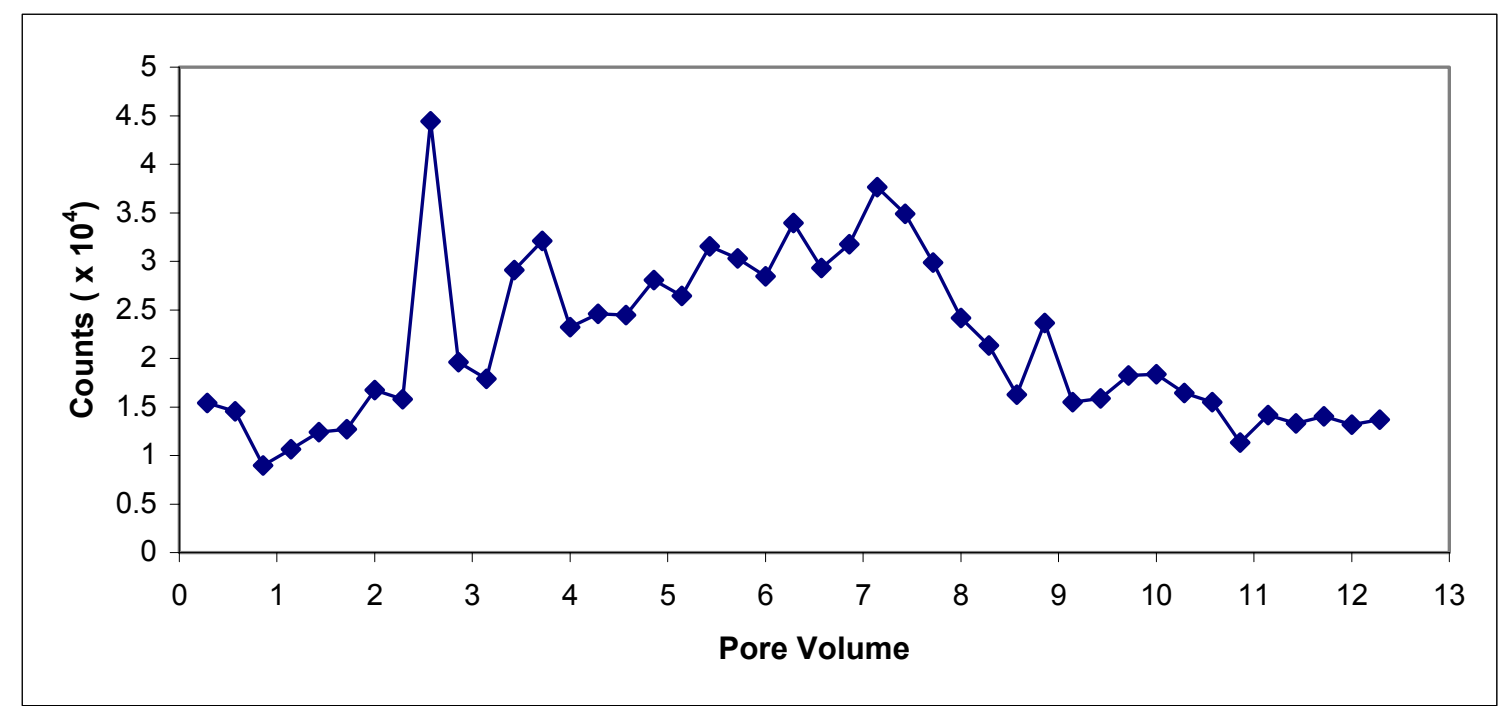

Figure 5.

This graph displays the fluorescence of the samples collected. 
The pore volume, which is the column's fluid capacity, was $35 \mathrm{~mL}$ in our experiment. The sharp peaks in the graph reflect high counts of the samples. The low counts at the beginning of the graph reflect that the pores did not contain colloids, or the colloids went undetected. The linear trend of the majority of the graph indicates injection of the colloids. Where the graph begins to dip downward reflects the rinsing of the column. The counts dropped at this point because the colloids had been purged from the column and only salt solution was being collected. The overall low counts of the samples led to the conclusion that the colloids did not pass through the column very well.

\section{CONCLUSIONS}

This project consisted of the synthesis of fluorescein-tagged PLA colloids, characterization of these colloids, and determination of the fraction of colloids that passed through a simulated ground system. Each process or experiment contributed to the goal of creating colloids that can eventually be used successfully for in situ bioremediation.

The synthesis portion of the project examined the parameters necessary for engineering colloids. The best methods for altering the size of colloids were determined from the many trials run and the variations in the methods used to create the colloids. From the data collected, future investigators will have a better idea of what improvements to make to in processing of colloids.

The characterization experiments investigated what properties the colloids displayed. The size, surface charge, and fluorescence of the colloids were determined in these experiments. The particle size analyzer displayed the difference in the size of the colloids before and after transport through the basalt column. The difference between the fluorescent spectra of the colloids and the unincorporated dye was also determined. Comparing the fluorescence of the colloids before and after filtration indicated the suspension of free, or unincorporated, dye in the colloidal suspension. From this development came a better understanding of fluorescence. This indication will be helpful in future determinations and applications of particle fluorescence.

The basalt column experiment examined how well the colloids were transported through the column. Particle size analysis and fluorescence spectrometry revealed the colloids' mobility. Only a small fraction of the particles passed through the basalt column. This indicated that the colloids were sticking somewhere in the column. Since basalt has nonporous surfaces, it is assumed that the colloids were getting stuck between the spaces among the basalt particles. This indicated that the colloids needed to be smaller. This conclusion can be applied to future research on colloidal suspensions.

Overall, the results and data from the experiments conducted produced information that can be applied to the processing and characterization of PLA colloids and for in situ bioremediation of groundwater. 


\section{ACKNOWLEDGMENT}

This work was supported through Mickey Leland Energy Fellowship (MLEF)

program and the INEEL Laboratory Directed Research and Development Program under DOE Idaho Operations Office Contract DE-AC07-99ID13727.

\section{REFERENCES}

1. Thomas, J.M. and Ward, C.H., "In situ biorestoration of organic contaminants in the Subsurface," Environmental Science and Technology, Volume 23, Number 7, 1989.

2. McCarthy, John F. and Zachara, John M., "Subsurface transport of contaminants," Environmental Science and Technology, Volume 23, Number 5, 1989.

3. Holland, S.J., Tighe, B.J. and Gould, P.L., "Polymers for Biodegradable Medical Devices, the Potential of Polyesters as Controlled Macromolecular Release Systems," Journal of Controlled Release, Volume 4, 1986, pp.155-180.

4. Lactic acid,

http://www.m-w.com/cgi-bin/dictionary?book=Dictionary\&va=polymer, Merriam Webster, Incorporated, 2003.

5. Kosmulski, Michal, About Zeta Potential - Electrokinetic Phenomena, http://zeta-potential.sourceforge.net/zeta-potential.shtml. 